\title{
VARIACIONES DIURNAS DE ESPESOR Y CURVATURA CORNEAL CENTRAL Y PARACENTRAL
}

\section{DIURNAL VARIATIONS OF CENTRAL AND PARACENTRAL CORNEAL THICKNESS AND CURVATURE}

GIRÁLDEZ-FERNÁNDEZ MJ ${ }^{1}$, DÍAZ-REY A², GARCÍA-RESUA C ${ }^{1}$, YEBRA-PIMENTEL-VILAR E ${ }^{3}$

\section{RESUMEN}

Objetivo: La topografía y espesor de la córnea tienen una gran importancia clínica en la adaptación de lentes de contacto y en cirugía refractiva. Sin embargo, la repetibilidad en las medidas de estos parámetros se ve comprometida por el fenómeno natural de las variaciones diurnas. Nuestro objetivo es determinar las variaciones diurnas de espesor y curvatura corneal central y paracentral durante un periodo de 10 horas.

Material y método: Se determinó la curvatura y espesor corneal en los ojos derechos de 10 varones jóvenes mediante Orbscan y videoqueratoscopio respectivamente. Ambos parámetros fueron determinados para la zona central y paracentral a 1 y 2 $\mathrm{mm}$ del centro de la córnea, cada dos horas durante un periodo de 10 horas.

Resultados: La córnea presentaba su espesor máximo y curvatura mínima al abrir el ojo. Se observó una variación estadísticamente significativa de espesor (ANOVA, método de Schefféc $\mathrm{p}<0,05$ ) y de curvatura (ANOVA, método de Tamhane $\mathrm{p}<$ $0,05)$ a lo largo del día en todas las localizaciones estudiadas, si bien la variación y los rangos de valores en la zona paracentral eran mayores. Los valo-
Objective: Corneal topography and thickness has clinical importance in contact lens fitting and refractive surgery, however repeated measurement of corneal thickness and curvature is complicated by the natural phenomenon of diurnal variation. Our aim was determine diurnal variations of central and paracentral corneal thickness and curvature over a period of ten hours.

Materials and methods: Corneal thickness and curvature of 10 right eyes of 10 young healthy men were determined by Orbscan Topography System and EyeSys videokeratoscope respectively. Both parameters were determined for central and paracentral regions $1 \mathrm{~mm}$ and $2 \mathrm{~mm}$ from the centre of the cornea at 2-hour internals for ten hours.

Results: The cornea was thickest and flattest on awakening. There was a difference in corneal thickness (ANOVA, Schfféc method $p<0.05$ ) and curvature (ANOVA, Tamhane method $\mathrm{p}<0.05$ ) over time for all the corneal locations studied, with greater changes observed in the peripheral corneal data. The minimum value was 10 and 8 hours after eye opening for central and paracentral zones, respectively. Change in central and paracentral corneal

\footnotetext{
Recibido: 29/1/07. Aceptado: 15/2/08.

Universidad de Santiago de Compostela. Santiago de Compostela. España.

1 Diplomado en Óptica y Optometría.

2 Licenciado en Medicina. Oftalmólogo. Universidad de Minho, Braga. Portugal.

${ }^{3}$ Doctora en Farmacia.

Correspondencia:

Eva Yebra-Pimentel Vilar

Escuela Universitaria de Óptica y Optometría

Universidad de Santiago de Compostela. Campus Sur

15782 Santiago de Compostela

España

E-mail: eyebra@usc.es
} 
res mínimos para ambos parámetros se observaron a las 10 y 8 horas después de abrir los ojos para las zonas central y paracentral respectivamente. Las variaciones de espesor y curvatura estaban altamente correlacionadas excepto para la localización nasal y superior a $2 \mathrm{~mm}$ del centro.

Conclusiones: Estos datos evidencian un cambio en espesor y curvatura que pueden ser de relevancia clínica atendiendo a la variación individual encontrada.

Palabras clave: Córnea, espesor corneal, curvatura corneal, paquimetría, variación diurna. thickness was strongly correlated with corneal curvature, except for the $2 \mathrm{~mm}$ nasal and superior semi-meridians.

Conclusions: These data indicate a shift in corneal thickness and curvature that can be of clinical relevance as individual changes vary greatly (Arch Soc Esp Oftalmol 2008; 83: 183-192).

Key words: Cornea, corneal thickness, corneal curvature, pachymetry, diurnal variation.

\section{INTRODUCCIÓN}

Conocer el espesor corneal ha adquirido gran relevancia en los últimos años, en parte, como consecuencia del creciente interés en el uso continuado de lentes de contacto $(1,2)$ y en cirugía refractiva (35). La medida del espesor corneal proporciona una información muy válida sobre el estado fisiológico de la córnea y sobre posibles cambios asociados a enfermedades, traumas e hipoxia.

Las variaciones diurnas del espesor corneal dificultan la repetitividad de las medidas paquimétricas. El patrón que sigue dicha variación es de máximo espesor en el momento de abrir los ojos después del cierre nocturno (6-8). Tanto la paquimetría óptica como la ultrasónica han sido utilizados para medir el espesor corneal en periodos de 12 a 48 horas (7-10). Kiely y col (7), utilizando el paquímetro óptico Haag-Streit, encuentra una variación diurna de $10 \mu \mathrm{m}$ para el espesor central y de $20 \mu \mathrm{m}$ en la periferia a $40^{\circ}$.

La curvatura corneal también ha sido considerada en relación con la cirugía refractiva, la adaptación de LC, etc. Son varios los autores que han estudiado el cambio diurno de la curvatura corneal central, que muestran que la córnea es más plana al despertarse, con un ligero, pero estadísticamente significativo, encurvamiento a lo largo del día $(6,10,11)$, observándose variaciones más significativas en los hemimerididanos superior e inferior (12) y diferencias de género (13).

Kiely y col (7) estudiaron la relación entre la curvatura y espesor corneal, encontrando una correlación significativa entre las variaciones diurnas de espesor y de curvatura en el meridiano horizontal. Por otro lado, Rom y col (14) no encuentran rela- ción entre las variaciones de espesor y de topografía corneal.

En este estudio se determinaron los valores de espesor y curvatura corneal central y paracentral (a 1 y a $2 \mathrm{~mm}$ del centro) durante un periodo de 10 horas, realizándose las medidas cada dos horas. La finalidad es determinar las variaciones diurnas de espesor y curvatura corneal en las localizaciones central y paracentral, y estudiar la relación entre las variaciones de espesor y curvatura.

\section{SUJETOS, MATERIAL Y MÉTODO}

En el estudio participaron diez hombres jóvenes, sin historia de uso de lentes de contacto, sin patología ocular o sistémica, y que no estaban recibiendo ningún tipo de medicación tópica o sistémica. No se incluyó en la muestra de estudio a ninguna mujer, con el fin de evitar los efectos hormonales sobre el espesor y curvatura corneal (15). Una vez que los procedimientos fueron explicados a los pacientes, éstos firmaron el consentimiento informado.

Inmediatamente al despertarse, y con la finalidad de eliminar el efecto de la evaporación de la lágrima sobre el deshinchamiento de la córnea (8), cada sujeto se tapaba su ojo derecho con un parche hasta el momento de la primera medida. Los valores de espesor y curvatura de la córnea del ojo derecho fueron determinados con el Orbscan Topography System (Orbscan II version 3.0) y con el EyeSys videokeratoscope (EyeSys Corneal Analisys System 2000, versión 3.1; EyeSys, INC, Houston, TX), respectivamente.

Aunque, estudios previos han demostrado que el Orbscan proporciona valores más altos que los 
paquímetros ópticos y ultrasónicos $(16,17)$, se ha demostrado su utilidad para la medida del espesor corneal en investigación y en clínica, con una precisión sobre bolas de calibrado de $\pm 2 \mu \mathrm{m}$ (rango, 1,2 $\mu \mathrm{m}$ en el centro a $3,2 \mu \mathrm{m}$ en la periferia) $(10,16)$. Medidas repetidas realizadas sobre personas, muestran una desviación estándar media de 9,08 um en los $7 \mathrm{~mm}$ centrales de la córnea (10). Sin embargo, la repetitividad y reproducibilidad del Orbscan es baja cuando se trata de medir la curvatura corneal (18), por lo que los valores de curvatura se determinaron mediante viedoqueratoscopía. Estudios previos muestran una alta repetitividadad en las medidas realizadas con el videoqueratoscopio, con una desviación estándar de $\pm 0,01 \mathrm{~mm}$ en personas y en bolas de calibrado $(19,20)$.

Las medidas de espesor y curvatura se tomaron en nueve localizaciones corneales: central y a 1 y 2 $\mathrm{mm}$ del centro en los hemimeridianos nasal, temporal, superior e inferior. Las primeras medidas se realizaron justo después de retirar el parche del ojo, y a partir de ese momento cada 2 horas durante un periodo de 10 horas.

El análisis estadístico se realizó utilizando el SPSS 6.1 (SPSS Inc., Chicago, Illinois, USA). Para comparar las variaciones diurnas de espesor y curvatura en las localizaciones estudiadas se aplicó un test ANOVA mediante un Modelo Lineal General Univariante. El espesor y la curvatura de la córnea fueron considerados como variables dependientes, mientras que la localización central, a 1 y a $2 \mathrm{~mm}$ del centro, meridianos y el momento de medida fueron considerados como los factores. Puesto que el test de Levene para la igualdad de las varianzas era estadísticamente significativo para el espesor y no para la curvatura, los métodos de Sheffec y de Tamhane fueron utilizados para espesor y curvatura respectivamente. La relación entre las medidas de espesor y curvatura se evaluó mediante un análisis de regresión lineal.

\section{RESULTADOS}

Los valores de espesor y curvatura corneal central y nasal, temporal, superior e inferior a 1 y $2 \mathrm{~mm}$ del centro de la córnea se muestran en la figura 1 y 2 respectivamente. El análisis estadístico mostró una variación en el espesor corneal estadísticamente significativa en todas las localizaciones estudiadas durante el periodo de estudio (ANOVA, método de Schfféc $p<0,05)$. La córnea presentaba su espesor máximo en el momento de abrir el ojo después del cierre nocturno y disminuía durante el día en todas las localizaciones analizadas. La variación media diurna en el espesor corneal central fue de 14 $\mu \mathrm{m}(1,95 \%)$, con valores individuales en un rango de 9 a $22 \mu \mathrm{m}$, y con el valor mínimo a las 10 horas de la apertura del ojo. La pendiente media del des-
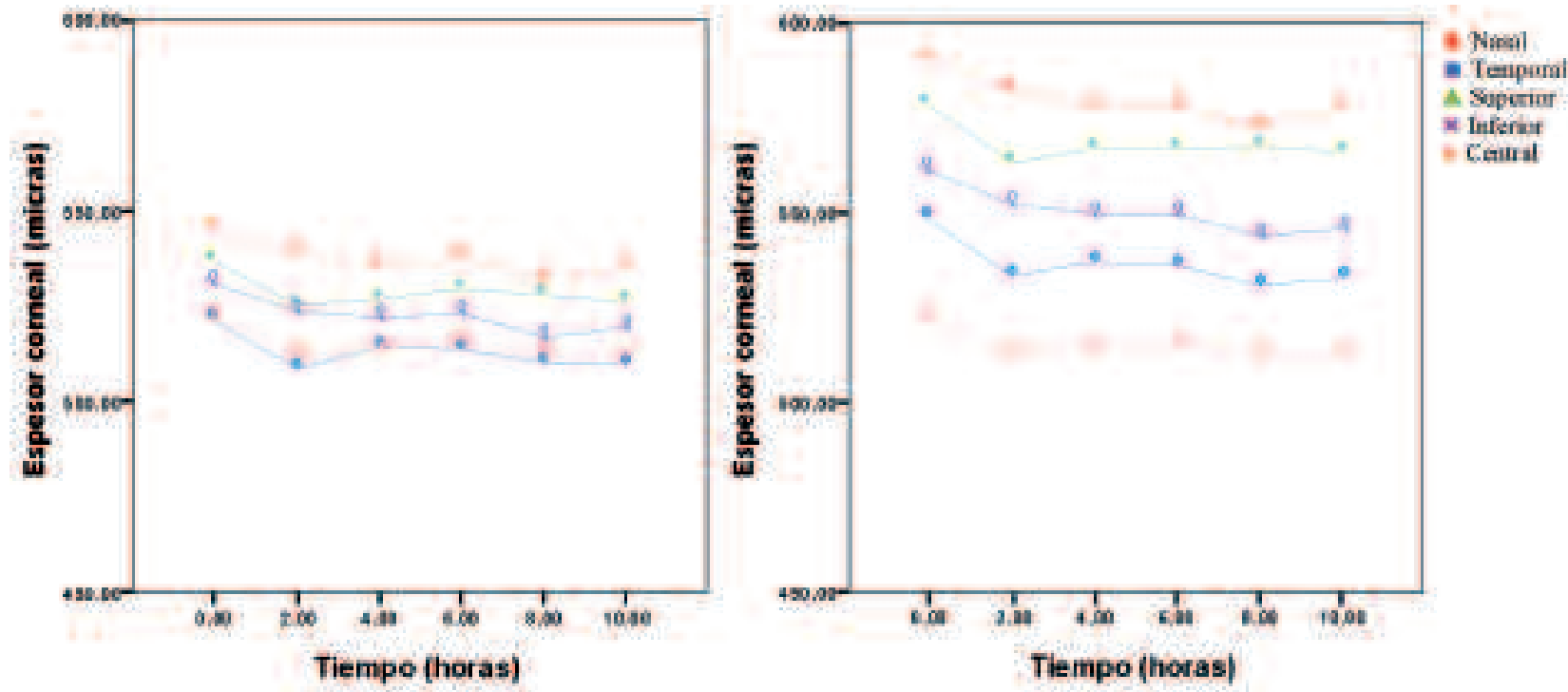

Fig. 1: Valor medio de espesor corneal central y nasal, temporal, superior e inferior inmediatamente después de abrir el ojo y cada dos horas durante un periodo de 10 horas a) $1 \mathrm{~mm}$ del centro de la cornea; b) $2 \mathrm{~mm}$ del centro de la córnea. 

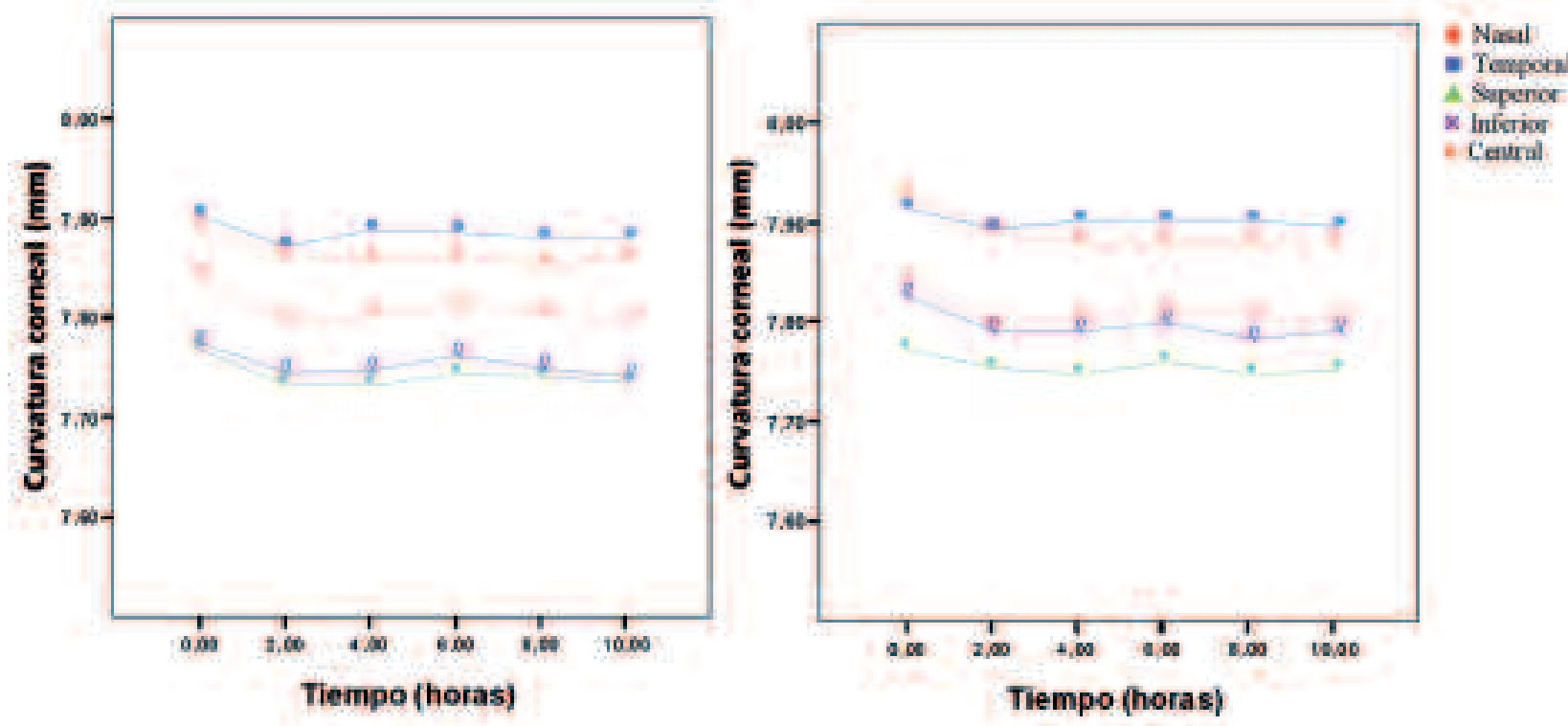

Fig. 2: Valor medio de curvatura corneal central central y nasal, temporal, superior e inferior inmediatamente después de abrir el ojo y cada dos horas durante un período de 10 horas a) $1 \mathrm{~mm}$ del centro de la cornea; b) $2 \mathrm{~mm}$ del centro de la córnea.

censo paquimétrico en el centro de la córnea fue de $-0,686$. Las variaciones de espesor correspondientes a las localizaciones nasal, temporal, superior e inferior a 1 y $2 \mathrm{~mm}$ del centro se muestran en las tablas I y II, encontrándose el valor mínimo sobre las 8 horas después de abrir el ojo. La pendiente media

Tabla I. Porcentaje de variación diurna máxima en el espesor corneal a 1 y $2 \mathrm{~mm}$ del centro en los hemimeridianos nasal, temporal, superior e inferior

\begin{tabular}{lcccc}
\hline $\begin{array}{l}\text { Localización } \\
\text { desde el centro }\end{array}$ & Nasal & Temporal & Superior & Inferior \\
\hline $1 \mathrm{~mm}(\%)$ & 2,23 & 2,57 & 2,13 & 2,70 \\
$2 \mathrm{~mm}(\%)$ & 2,97 & 2,80 & 2,66 & 3,14 \\
\hline \hline
\end{tabular}

Tabla II. Cambio en el espesor corneal a 1 y 2 mm del centro en los hemimeridianos nasal, temporal, superior e inferior, durante un período de $\mathbf{1 0}$ horas después de abrir los ojos (valor medio y rango de valores)

\begin{tabular}{lcccc}
\hline $\begin{array}{l}\text { Localización } \\
\text { desde el centro }\end{array}$ & Nasal & Temporal & Superior & Inferior \\
\hline $1 \mathrm{~mm}(\mu \mathrm{m})$ & $22(7-32)$ & $22(13-31)$ & $24(12-27)$ & $20(8-26)$ \\
$2 \mathrm{~mm}(\mu \mathrm{m})$ & $19(10-40)$ & $19(12-47)$ & $23(12-36)$ & $16(13-44)$ \\
\hline \hline
\end{tabular}

del descenso paquimétrico correspondiente a 1 y 2 $\mathrm{mm}$ en los meridianos estudiados se muestran en la tabla III, observándose que los valores correspondientes a las zonas paracentrales nasal, temporal, inferior y superior a $2 \mathrm{~mm}$ indican una disminución paquimétrica mayor que en el centro de la córnea $(-0,686)$. En el caso del meridiano superior, puede verse que la pendiente media de la variación de espesor a $1 \mathrm{~mm}$ del centro es ligeramente inferior al central, y el valor obtenido a $2 \mathrm{~mm}$, aunque mayor, es el que más se aproxima a la variación paquimétrica en el centro de la córnea. La menor curvatura se observó al abrir el ojo después del cierre nocturno, con una diferencia estadísticamente significativa cuando se compara con los valores sucesivos obtenidos a lo largo del día (ANOVA, método de Tamhane $\mathrm{p}<0,05)$. Después de un aumento inicial de la curvatura corneal, ésta permanece práctica-

Tabla III. Pendiente media del descenso paquimétrico en las localizaciones paracentrales a 1 y $2 \mathrm{~mm}$ del centro en los hemimeridianos nasal, temporal, superior e inferior

\begin{tabular}{lcccc}
\hline $\begin{array}{l}\text { Localización } \\
\text { desde el centro }\end{array}$ & Nasal & Temporal & Superior & Inferior \\
\hline $1 \mathrm{~mm}(\mu \mathrm{m})$ & $-0,994$ & $-0,784$ & $-0,603$ & $-1,100$ \\
$2 \mathrm{~mm}(\mu \mathrm{m})$ & $-1,250$ & $-1,247$ & $-0,726$ & $-1,426$ \\
\hline \hline
\end{tabular}


mente estable a lo largo del día en todas las localizaciones estudiadas.

La variación media de curvatura central observada en el periodo de estudio fue de $0,07 \mathrm{~mm}$, con el valor mínimo a las 2 horas de abrir los ojos. En la tabla IV se presentan los valores correspondientes a las localizaciones paracentrales estudiadas.

No existía una diferencia estadísticamente significativa entre las variaciones de espesor (ANOVA, método de Schefféc $\mathrm{p}>0,05)$ así como de curvatura (ANOVA, método de Tamhane $\mathrm{p}>0,05$ ) observadas en el centro de la córnea, a 1 y $2 \mathrm{~mm}$ del centro en todos los hemimeridianos medidos. De manera similar, tampoco se observaron diferencias estadísticamente significativas entre las variaciones de espesor (ANOVA, método de Schefféc $\mathrm{p}>0,05$ ) ni entre las variaciones de curvatura (ANOVA, método de Tamhane $\mathrm{p}>0,05$ ) que tienen lugar en los hemimeridianos corneales estudiados. Las variaciones de espesor y curvatura nasal, temporal, superior e inferior a 1 y $2 \mathrm{~mm}$ del centro se muestran en la figura 3 y 4 respectivamente.

En la tabla V se presenta los resultados del análisis estadístico en el que se comparan las variaciones diurnas de espesor y curvatura en todas las localizaciones corneales estudiadas. Se observó un alto grado de correlación entre las variaciones en el centro de la córnea $\left(r=0,983, r^{2}=0,967, p<0,001\right)$ y en las demás localizaciones, excepto para la zona nasal y superior a $2 \mathrm{~mm}$ del centro.

Tabla V. Correlación entre las variaciones diurnas de espesor y curvatura en cada localización estudiada

\begin{tabular}{llccc}
\hline & & $\mathrm{r}$ & $\mathrm{r}^{2}$ & $\mathrm{p}$ \\
\hline Nasal & $1 \mathrm{~mm}$ & 0,933 & 0,870 & 0,007 \\
& $2 \mathrm{~mm}$ & 0,604 & 0,365 & 0,204 \\
Temporal & $1 \mathrm{~mm}$ & 0,968 & 0,937 & 0,002 \\
& $2 \mathrm{~mm}$ & 0,848 & 0,719 & 0,033 \\
Superior & $1 \mathrm{~mm}$ & 0,991 & 0,982 & 0,000 \\
& $2 \mathrm{~mm}$ & 0,797 & 0,635 & 0,058 \\
Inferior & $1 \mathrm{~mm}$ & 0,817 & 0,667 & 0,047 \\
& $2 \mathrm{~mm}$ & 0,927 & 0,859 & 0,008 \\
\hline \hline
\end{tabular}

\section{DISCUSIÓN}

La finalidad de este trabajo es profundizar en el conocimiento del efecto que tiene el tiempo sobre el espesor y curvatura corneal a lo largo del día. Determinando si existen cambios en el espesor y curvatura corneal central y paracentral a lo largo del tiempo, podríamos establecer el momento en el que se deberían tomar medidas basales.

La relevancia e implicaciones de conocer las variaciones diurnas de espesor corneal son particularmente importantes en la evaluación clínica de pacientes de PRK y en candidatos a otras cirugías queratorefractivas. De hecho, la contraindicación más común para LASIK es un espesor corneal central reducido, siendo también un factor importante en las características del colgajo creado durante el procedimiento $(21,22)$. La curvatura de la córnea también ha sido considerada en relación con la cirugía refractiva por su relación con el espesor y diámetro del colgajo (23).

En este estudio, el espesor corneal máximo era observado inmediatamente después de abrir el ojo por la mañana en todas las localizaciones estudiadas, hallazgo que coincide con otros estudios (510). La disminución de aporte de oxígeno que tiene lugar en el cierre ocular nocturno lleva a un metabolismo anaeróbico que produce un aumento de ácido láctico. Como consecuencia se produce un aumento de la presión osmótica que favorece la difusión de agua desde el humor acuoso hacia el estroma, con el consecuente aumento de espesor corneal (24). Cuando se compara el espesor corneal obtenido durante un periodo de 10 horas, observamos una variación estadísticamente significativa en todas las localizaciones estudiadas. Aunque la variación media diurna del espesor corneal central observada $(14 \mu \mathrm{m})$ fue similar a la encontrada por otros autores $(7,10,25)$ las variaciones individuales pueden llegar a ser clínicamente relevantes (rango de 9 a $22 \mu \mathrm{m}$ ). Como puede verse en la tabla II la magnitud de cambio y el rango de valores en el espesor es mayor en los datos paracentrales, hecho

Tabla IV. Variación media y rango de valores de la curvatura central y paracentral a 1 y 2 mm del centro en los hemimeridianos nasal, temporal, superior e inferior $(\mathbf{m m})$

\begin{tabular}{|c|c|c|c|c|c|}
\hline Central & Localización & Nasal & Temporal & Superior & Inferior \\
\hline \multirow[t]{2}{*}{$0,07(0,05-0,1)$} & $1 \mathrm{~mm}$ & $0,05(0,01-0,10)$ & $0,06(0,03-0,11)$ & $0,07(0,03-0,11)$ & $0,06(0,03-0,12)$ \\
\hline & $2 \mathrm{~mm}$ & $0,07(0,02-0,12)$ & $0,07(0,02-0,16)$ & $0,06(0,03-0,20)$ & $0,07(0,05-0,13)$ \\
\hline
\end{tabular}



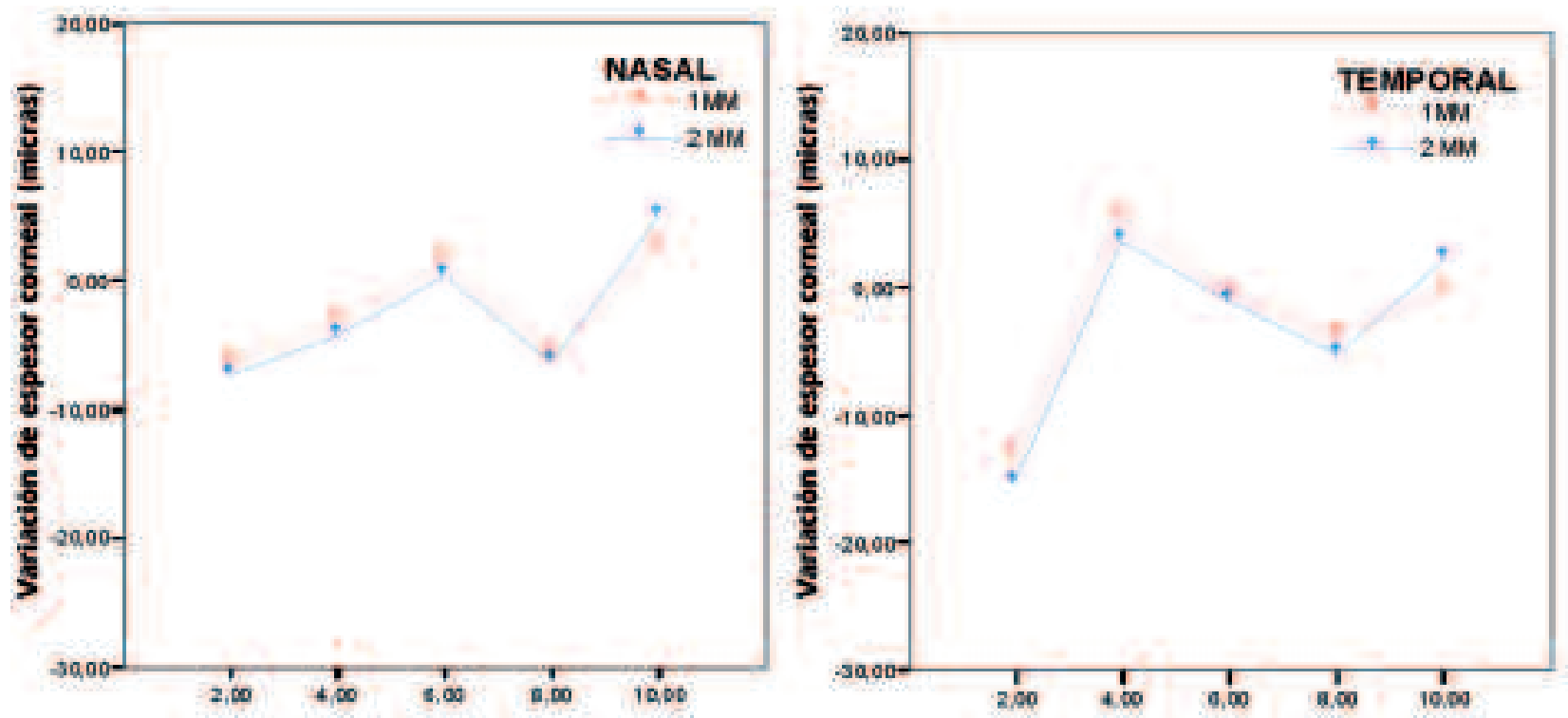

Tiempo (horas)
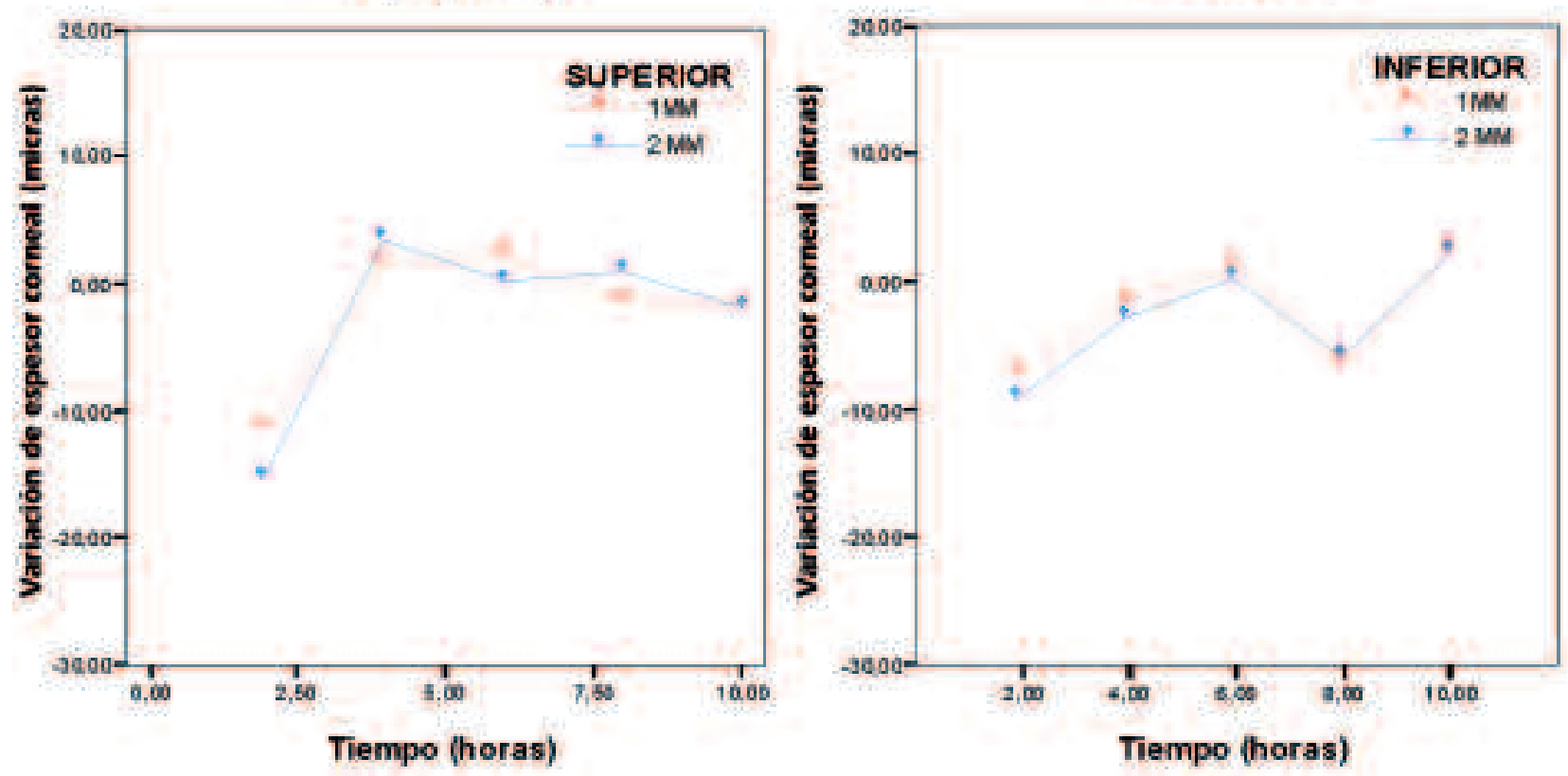

Fig. 3: Variación de espesor corneal a 1 y $2 \mathrm{~mm}$ del centro en los hemimeridianos nasal, temporal, superior e inferior.

que también se pone de manifiesto al comparar los valores de pendiente media del descenso paquimétrico central $(-0,686)$ con los valores correspondientes a las zonas paracentrales nasal, temporal, inferior y superior a $2 \mathrm{~mm}$ (tabla III). En el caso del meridiano superior, puede verse que la pendiente media de la variación de espesor a $1 \mathrm{~mm}$ del centro es ligeramente inferior al central, y el valor obteni- do a $2 \mathrm{~mm}$, aunque mayor, es el que más se aproxima al valor central. Este hecho puede deberse a que la córnea superior, tanto en condiciones de ojo cerrado como abierto, es la zona con mayor estress hipóxico, lo que le lleva a una menor disminución en el espesor en condiciones de ojo abierto (26-28). El incremento de espesor hacia la periferia de la córnea y la mayor variación de espesor que se 

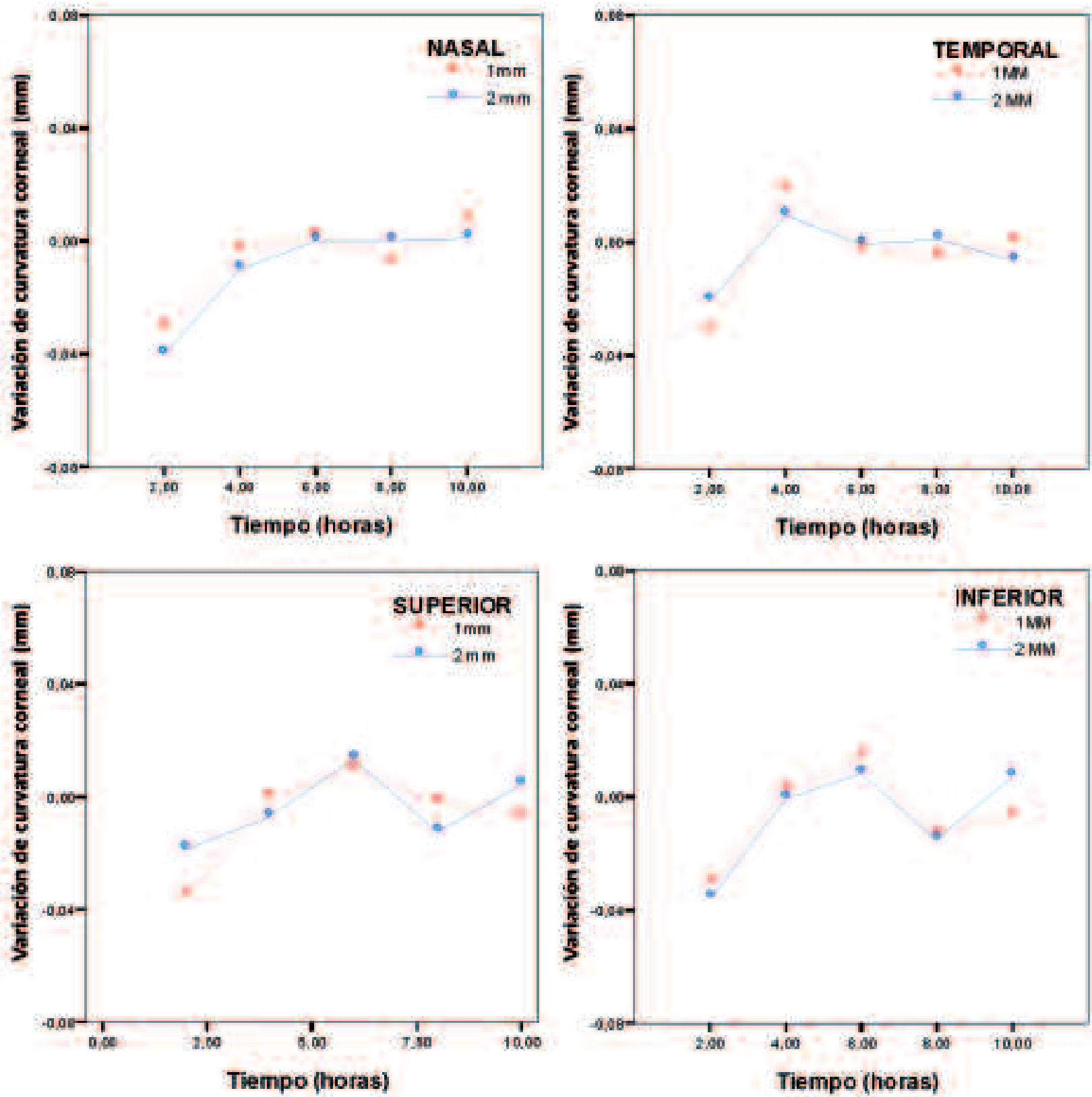

Fig. 4: Variación de curvatura corneal a 1 y 2 mm del centro en los hemimeridianos nasal, temporal, superior e inferior.

observa, podría denotar un cambio tanto en la capacidad reguladora de la hidratación como en su control específico, indicando la presencia de un gradiente lateral de hidratación que se extiende desde el ápex a la periferia (10). La variación de hidratación a lo largo del perfil de la córnea puede ser una fuente de error en algunos pacientes de cirugía refractiva, puesto que la variación diurna en la paquimetría podría llegar a ser mayor que la cantidad de tejido eliminado por dioptría. Además, muchos microqueratomos generan meniscos de grosor no uniforme, de manera que la tensión biomecánica corneal podría verse comprometida si en determinadas localizaciones el estroma residual es muy bajo. Si el valor de mínimo espesor corneal no se determina adecuadamente, las medidas pueden 
teóricamente resultar en un falso valor mayor no real, llevando a una sobreestimación que puede incrementar el riesgo de desarrollo de querectasia postoperativa.

El espesor corneal central y paracentral presentaban su valor mínimo a las 10 y 8 horas después de abrir los ojos respectivamente. Estudios previos han demostrado que los valores más bajos de espesor corneal se obtienen entre las 5 y 12 horas después de abrir el ojo (7-9,25), obteniéndose, en casos, un adelgazamiento corneal que supera el valor basal. Las diferencias entre estudios puede deberse a diferencias en los intervalos y frecuencia de medida. El adelgazamiento de la córnea más allá del valor basal ha sido estudiado previamente (29), y si bien su presencia está demostrada, es un aspecto de la dinámica de hidratación corneal al que todavía no se le ha encontrado una explicación. Odenthal y col (29) sugiere como más probable que la velocidad de la bomba endotelial no es constante, y que un estrés hipóxico puede activar una reserva funcional en la capacidad de la bomba iónica, que retrase su actividad a un nivel normal. Cuando esta situación se produce, el espesor corneal podría verse influido por otros factores, tales como la frecuencia del parpadeo, la calidad de la película lagrimal, y variaciones diurnas en la producción de lágrima y en la presión intraocular, lo que podría explicar el incremento de espesor que se produce tras alcanzar la córnea su espesor mínimo entre las 8 y 10 horas del cierre ocular nocturno (29).

En lo que a curvatura se refiere, nuestros resultados son consistentes con los encontrados por otros investigadores $(7,11,13)$. Inicialmente las córneas presentan el valor más plano, y a lo largo del día aumenta la curvatura, con mínimas fluctuaciones. Sin embargo, en contraste con Kiely y col (7), no observamos diferencias en las variaciones diurnas que tienen lugar en los meridianos horizontal y vertical. El trabajo de cerca y la presión de los párpados pueden tener un papel relevante en los cambios diurnos de la curvatura corneal $(12,30,31)$, por lo que es probable que los resultados en este estudio difieran de los encontrados en otros, en los que los sujetos hubiesen realizado tareas en cerca durante el periodo de medida. Además, en nuestro estudio el efecto de los párpados es poco probable cuando las medidas se realizan a $1 \mathrm{y}$ $2 \mathrm{~mm}$ del centro de la córnea. Read y col (12) sugiere que existe una correlación entre la cantidad de trabajo en visión próxima realizado y el grado de cambio de curvatura, siendo posibles factores de influencia la hidratación corneal, la estabilidad de la lágrima, la frecuencia del parpadeo, la apertura palpebral vertical y el tiempo transcurrido entre el momento de trabajo en cerca y el de medida. Aunque no se observaron diferencias estadísticamente significativas entre meridianos, en la figura 4 se puede observar un incremento de curvatura en los meridianos superior e inferior a las 8 horas de abrir los ojos. Este hallazgo coincide con otros estudios que refieren un incremento en la curvatura en el meridiano vertical a última hora del día $(11,12)$.

Se ha sugerido que cambios diurnos en la curvatura corneal en ojos normales puede estar relacionado con cambios en el espesor de la córnea $(7,32)$. MacRae y col (32) encontraron que en ojos normales y en ojos tratados de queratotomía radial existía una relación entre el espesor corneal y el cambio en la forma de la córnea. Sin embargo, observaron que tal correlación no era estadísticamente significativa cuando la muestra se dividía en tres grupos (8 pacientes por grupo). En nuestro estudio el análisis estadístico muestra una fuerte correlación entre los cambios de espesor y curvatura corneal (tabla V). Estos resultados pueden ser gráficamente observados en la similitud existente entre las figuras 2 y 4 , y concuerdan con otros estudios que incluyen córneas normales y tratadas de queratotomía radial $(32,33)$. Por otro lado Kwitko y col (34), no observan tal relación y consideran que se debe al pequeño número de sujetos que forman su muestra, que limita el análisis estadístico y la detección de correlaciones estadísticas. Sorprendentemente con el mismo tamaño de muestra que Kwitko y col (34), nosotros alcanzamos significación estadística con una tendencia similar a la encontrada por MacRae y col (32), en la que cuando el espesor de la córnea disminuye, la curvatura aumenta. Esta relación entre espesor y curvatura podría deberse a la mayor disminución de espesor que tiene lugar en la zona paracentral de la córnea, cuando se compara con el centro de la misma, es decir, dicha diferencia en la disminución de espesor, podría ser la causa del encurvamiento observado.

\section{BIBLIOGRAFÍA}

1. Brennan NA, Coles ML. Extended wear in perspective. Optom Vis Sci 1997; 74: 609-623. 
2. Yebra-Pimentel E, Giraldez MJ, Gonzalez J, Perez MV, Parafita M. Changes in corneal thickness after daily and extended wear of hydrogel lenses: a comparison of optical and ultrasonic pachometry. Int Contact Lens Clin 1998; 25: 103-107.

3. Pallikaris IG, Kymionis GD, Astyrakakis NI. Corneal ectasia induced by laser in situ keratomileusis. J Cataract Refract Surg 2001; 27: 1796-1802.

4. Rao SN, Epstein RJ. Early onset ectasia following laser in situ keratomileusis: case report and literature review. J Refract Surg 2002; 18: 177-184.

5. Jonsson M, Behndig A. Pachymetric evaluation prior to laser in situ keratomileusis. J Cataract Refract Surg 2005; 31: 701-706.

6. Mertz GW. Overnight swelling of the living human cornea. J Am Optom Assoc 1980; 51: 211-214.

7. Kiely PM, Carney LG, Smith G. Diurnal variations of corneal topography and thickness. Am J Optom Physiol Opt 1982; 59: 976-982.

8. Holden BA, Mertz GW, McNally JJ. Corneal swelling response to contact lenses worn under extended wear conditions. Invest Ophthalmol Vis Sci 1983; 24: 218-226.

9. Harper CL, Boulton ME, Bennet D, Marcyniuk B, JarvisEvans JH, Tullos AB, et al. Diurnal variations in human corneal thickness. Br J Ophthalmol 1996; 80: 1068-1072.

10. Lattimore MR Jr, Kaupp S, Schallhorn S, Lewis $R$ 4th. Orbscan pachymetry: implications of a repeated measures and diurnal variation analysis. Ophthalmology 1999; 106: 977-981.

11. Cronje S, Harris WF. Short-term keratometric variation in the human eye. Optom Vis Sci 1997; 74: 420-424.

12. Read SA, Collins MJ, Carney LG. The diurnal variation of corneal topography and aberrations. Cornea 2005; 24: 678-687.

13. Handa T, Mukuno K, Niida T, Uozato H, Tanaka S, Shimizu K. Diurnal variation of human corneal curvature in young adults. J Refract Surg 2002; 18: 58-62.

14. Rom ME, Keller WB, Meyer CJ, Meisler DM, Chern KC, Lowder CY, et al. Relationship between corneal edema and topography. CLAO J 1995; 21: 191-194.

15. Kiely PM, Carney LG, Smith G. Menstrual cycle variations of corneal topography and thickness. Am J Optom Physiol Opt 1983; 60: 822-829.

16. Marsich $M W$, Bullimore MA. The repeatability of corneal thickness measures. Cornea 2000; 19: 792-795.

17. Giraldez Fernandez MJ, Diaz, Rey A, Cerviño A, YebraPimentel E. A comparison of two pachymetric systems: silt-scanning and ultrasonic. CLAO J 2002; 28: 221-223.

18. Cho P, Lam AK, Mountford J, Ng L. The performance of four different corneal topographers on normal human corneas and its impact on orthokeratology lens fitting. Optom Vis Sci 2002; 79: 175-183.
19. Dave T, Ruston D, Fowler C. Evaluation of the EyeSys model II computerized videokeratoscope. Part I: Clinical assessment. Optom Vis Sci 1998; 75: 647-55.

20. Dave, T., Ruston, D. and Fowler, C. Evaluation of the EyeSys model II computerized videokeratoscope. Part II: The repeatability and accuracy in measuring convex aspheric surfaces. Optom Vis Sci 1998; 75: 656-662.

21. Miyata K, Tokunaga T, Nakahara M, Ohtani S, Nejima R, Kiuchi T, et al. Residual bed thickness and corneal forward shift after laser in situ keratomileusis. J Cataract Refract Surg 2004; 30: 1067-1072.

22. Ozdamar A, Kucuksumer Y, Devranoglu K. Flap dimensions created with the Hansatome microkeratome: correlation with Orbscan-derived anterior segment data. J Cataract Refract Surg 2005; 31: 1614-1617.

23. Spadea L, Cerrone L, Necozione S, Balestrazzi E. Flap measurements with the Hansatome microkeratome. J Refract Surg 2002; 18: 149-154.

24. Klyce SD. Stromal lactate accumulation can account for corenal oedema osmotically following wpithelial hypoxia in the rabbit. Journal of Physiology 1981; 321: 49-64.

25. du Toit R, Vega JA, Fonn D, Simpson T. Diurnal variation of corneal sensitivity and thickness. Cornea 2003; 22: 205-209.

26. Benjamin WJ, Rasmussen MA. Oxygen consumption of the superior cornea following eyelid closure. Acta Ophthalmol 1988; 66: 309-312.

27. Benjamin WJ, Ruben CM. Human corneal oxygen demands at superior, central and inferior sites. $J$ Am Optom Assoc 1995; 66: 423-428.

28. Erickson P, Comstock TL, Zantos SG. Effects of hydrogel lens transmissibility profiles on local corneal swelling during eye closure. Optom Vis Sci 1996; 73: 169-177.

29. Odenthal MT, Nieuwendaal CP, Venema HW, Oosting J, Kok JHC, Kijlstra A. In vivo human corneal hydration control dynamics: a new model. Invest Ophthalmol Vis Sci 1999; 40: 312-319.

30. Buehren T, Collins MJ, Carney L. Corneal aberrations and reading. Optom Vis Sci 2003; 80: 159-166.

31. Lieberman DM, Grierson JW. The lids influence on corneal shape. Cornea 2000; 19: 336-342.

32. MacRae S, Rich L, Phillips D, Bedrossian R. Diurnal variation in vision after radial keratotomy. Am J Ophthalmol 1989; 107: 262-267.

33. Kwitko S, Garbus JJ, Hwang DG, Gauderman WJ, McDonnell PJ. Computer-assisted study of diurnal variation in corneal topography after penetrating keratoplasty. Ophthalmic Surg 1992; 23: 10-16.

34. Kwitko S, Gritz DC, Garbus JJ, Gauderman WJ, McDonnell PJ. Diurnal variation of corneal topography after radial keratotomy. Arch Ophthalmol 1992; 110: 351356. 